\title{
ON A CONTINUOUS ANALOG OF SPERNER'S PROBLEM
}

\author{
L. H. HARPER \\ In memory of Ernst Straus
}

\begin{abstract}
An approach to Rota's question about the lattice of partitions is presented. Calculations, based upon the assumption that the lattice of partitions may be approximated by a Gaussian process, indicate that the ratio of the cardinality of the largest set of incomparable partitions to the largest rank is at least $1 / \sqrt{1-3 \sqrt{3} / 8} \simeq 1.69$ as $n \rightarrow \infty$.
\end{abstract}

0. Introduction. In 1928 E. Sperner solved the following problem: What is the largest size of any family, $I$, of subsets of an $n$-set such that no member of the family contains any other member? Such a family has been called "a family of incomparables" and Sperner's result was that max $|I|$ $=\left(\begin{array}{c}n \\ {[n / 2]}\end{array}\right)$, the largest binomial coefficient of order $n$.

The question which Sperner answered for the set of all subsets of an $n$-set, ordered by containment can easily be extended to any finite partial order $P$ having a rank function $r$. For subsets of an $n$-set the partial order is given by containment and the rank function by cardinality. Clearly no two elements of the same rank can be comparable in the partial order so $P_{k}=\{x \in P: r(x)=k\}$ is a family of incomparables. Sperner showed that in the case of subsets the lower bound $\max |I| \geq \max \left|P_{k}\right|$ is sharp. In 1970 G. C. Rota asked if this is also true for the set, $\Pi_{n}$, of all partitions of an $n$-set partially ordered by refinement. The rank of a partition is again its cadinality, i.e. the number of nonempty subsets (blocks) which it contains. Also $S_{n, k}$, the number of partitions of an $n$-set having $k$ blocks, is called a Stirling number of the second kind. As before, $\max _{I \subseteq \Pi_{n}}|I| \geq$ $\max _{k} S_{n, k}$ and Rota's question was whether the inequality is sharp. The question was an appealing one and there was considerable evidence that its answer was positive (e.g. it was shown to be true for $n \leq 19$ (see [7])) so enthusiasts began calling it "The Rota Conjecture". In 1976 however, E. R. Canfield found a counterexample to the Rota conjecture. Canfield showed that for $n \geq 6.5 \times 10^{24}$ you could take the largest rank, cut a relatively small piece out of it, replace that piece by a larger piece from the next rank and still have a family of incomparables. G. W. Peck (see 
[11]) sharpened Canfield's result to give counterexamples for $n \geq 5.5 \times$ $10^{6}$. However, none of these counterexamples gave a ratio of $\max |I|$ to $\max _{k} S_{n, k}$ greater than 1 in the limit. The assertion that the limit was indeed 1 became known, naturally, as "The Asymptotic Rota Conjecture".

The purpose of this paper is to present an insight into the lattice of partitions and calculations based upon it which suggest that

(i) The Rota Conjecture is essentially true for $n \leq 5.5 \times 10^{6}$ and

(ii) The asymptotic Rota conjecture is false, i.e. $\max |I| / \max S_{n, k}$ does not go to 1 as $n \rightarrow \infty$.

Because the argument is incomplete and takes some unexpected turns it will be presented organically, i.e. in the way it grew from the original insight.

I. Preliminaries. Our approach to the Asymptotic Rota Conjecture is based upon two statements, one a proven fact and another which, though a variant of published results, must still be regarded as a hypothesis. The first statement, the proven one, has been used in every paper on Rota's problem. It is that the action of $S_{n}$ the symmetric group on the underlying $n$-set, may be factored out of $\Pi_{n} \cdot \Pi_{n} / \mathbf{S}_{n}$ is the weighted partial order whose elements are $n$-tuples, $\sigma=\left(\sigma_{1}, \ldots, \sigma_{n}\right)$, of non-negative integers such that $\sum_{i=1}^{n} i \sigma_{i}=n$. $\sigma$ represents the equivalence class of all partitions having $\sigma_{i}$ blocks of size $i$ for $i=1$ to $n$. For $\sigma, \tau \in \Pi_{u} / \mathbf{S}_{n}$, $\sigma \leq \tau$ iff there exists a function $\alpha \geq 0$ such that

$$
\tau=\sigma+\sum_{i, j} \alpha(i, j)\left[\delta_{i}+\delta_{j}-\delta_{i+j}\right]
$$

where $\delta_{i}$ is the $n$-tuple whose $i$ th entry is 1 and all the rest are $0 . \Pi_{n}$ then has a minimum element $(0,0, \ldots, 0,1)$ and maximum element $(n, 0, \ldots, 0)$. The rank of $\sigma$ is $r(\sigma)=\sum_{i=1}^{n} \sigma_{i}$. The weight of $\sigma$ is

$$
W(\sigma)=n ! / \prod_{i=1}^{n} \sigma_{i} !(i !)^{\sigma_{i}}
$$

which is the number of partitions in the equivalence class of $\sigma$. Precisely speaking, the statement which we use is that the maximum weight of any set of incomparables in $\Pi_{n} / \mathbf{S}_{n}$ is equal to the maximum cardinality of any set of incomparables in $\Pi_{n}$. Rota himself suggested this means of reducing the problem; it was first given mathematical substance by R. L. Graham and subsequently developed by the present author (see [8]).

In light of the above statement we need only solve the weighted version of Sperner's problem on $\Pi_{n} / \mathbf{S}_{n}$. Now $B_{n}=\sum_{k=1}^{n} S_{n, k}$ is the 
number of partitions of an $n$-set, so if we divide the weight on $\sigma$ by $B_{n}$ we get a probability measure on $\Pi_{n} / \mathbf{S}_{n}, P(\sigma)=W(\sigma) / B_{n}$. The second statement, the hypothesis, is that this probability measure is asymptotically Gaussian (see [9]). To be more precise we mean that if $i$ is transformed to $(i-\lambda) / \gamma=s$ where $\lambda e^{\lambda}=n, \gamma^{2}=\lambda$ and $\sigma_{i}$ is transformed to $\left(\sigma_{i}-E\left(\sigma_{i}\right)\right) \lambda / \sqrt{n}=u_{s}^{(n)}$, then $u_{s}^{(n)}$ converges weakly to a Gaussian process $u_{s}$ having mean $E\left(u_{s}\right)=0$ and the appropriate covariance (calculated in the following section).

II. A continuous analog of the lattice of partitions. Let us expand a bit on the statement in the previous paragraph: As $i$ is transformed to the continuous variable $s$ (in the limit as $n \rightarrow \infty$ ) and $\sigma$ to the function $u$, then the rank of $\sigma, r(\sigma)=\sum_{i=1}^{n} \sigma_{i}$, is transformed to $r(u)=\int_{-\infty}^{\infty} u_{s} d s$ and the identity $\sum_{i=1}^{n} i \sigma_{i}=n$ to $\int_{-\infty}^{\infty}(s+\gamma) u_{s} d s=0$. Actually $\gamma \rightarrow \infty$ as $n \rightarrow \infty$, but very slowly (about like $\sqrt{\ln n}$ ) and must be retained as a parameter. The partial order on functions is characterized by $u \leq w$ iff there exists a function $\alpha \geq 0$ such that

$$
w=u+\int_{-\infty}^{\infty} \int_{-\infty}^{\infty} \alpha(x, y)\left[\delta_{x}+\delta_{y}-\delta_{x+y+\gamma}\right] d x d y
$$

where $\delta_{x}$ is a Dirac delta function.

Note that this partial order on the functions is given by a cone whose extreme points are $\left\{\delta_{x}+\delta_{y}-\delta_{x+y+\gamma}: x, y \in \mathbf{R}\right\}$. It is thus homogeneous which contrasts sharply with the orders of $\Pi_{n}$ and $\Pi_{n} / \mathbf{S}_{n}$. Note also that

$$
r(w)=r(u)+\int_{-\infty}^{\infty} \int_{-\infty}^{\infty} \alpha(x, y) d x d y .
$$

Gaussian distributions are characterized by their covariance functions so we must calculate $\operatorname{Cov}\left(\sigma_{r}, \sigma_{i j}\right)$ and estimate it as $n \rightarrow \infty$ :

LEMMA 1.

$$
\operatorname{Cov}\left(\sigma_{i}, \sigma_{j}\right)=\left(\begin{array}{c}
n \\
i
\end{array}\right) \frac{B_{n-i}}{B_{n}}\left[\delta_{i, j}+\left(\begin{array}{c}
n-i \\
j
\end{array}\right) \frac{B_{n-i-j}}{B_{n-i}}-\left(\begin{array}{c}
n \\
j
\end{array}\right) \frac{B_{n-j}}{B_{n}}\right]
$$

where

$$
\delta_{t, j}= \begin{cases}1 & \text { if } i=j \\ 0 & \text { otherwise }\end{cases}
$$


Proof. All moments of $\sigma$ may be computed by the same trick we use here to compute the mean:

$$
\begin{aligned}
& E\left(\sigma_{i}\right)=\frac{1}{B_{n}} \sum_{\pi \in \Pi_{n}} \sigma_{i}(\pi) \quad \text { where } B_{n}=\sum_{k=1}^{n} S_{n, k} \\
& =\frac{1}{B_{n}} \sum_{\pi \in \Pi_{n}} \sum_{\substack{|A|=i \\
A \subseteq\{1, \ldots, n\}}} X(\pi, A), \quad \text { where } X(\pi, A)= \begin{cases}1 & \text { if } A \in \pi, \\
0 & \text { if } A \notin \pi\end{cases} \\
& =\frac{1}{B_{n}} \sum_{|A|=i} \sum_{\pi \in \Pi_{n}} X(\pi, A)=\frac{1}{B_{n}} \sum_{|A|=i} B_{n-i}=\left(\begin{array}{c}
n \\
i
\end{array}\right) \frac{B_{n-i}}{B_{n}} .
\end{aligned}
$$

\section{LEMMA 2.}

$$
\left(\begin{array}{c}
n \\
i
\end{array}\right) \frac{B_{n-l}}{B_{n}}=\frac{\lambda_{n}^{i}}{i !}\left(1+O\left(\frac{\lambda_{n}^{2}}{n}\right)\right)
$$

where $\lambda_{n}$ is the solution of $\lambda e^{\lambda}=n$ and $i=O\left(\lambda_{n}\right)$.

Proof. Using

(i) Stirling's formula, $n !=\sqrt{2 \pi n}(n / e)^{n}(1+O(1 / n))$,

(ii) Moser \& Wyman's formula

$$
B_{n}=\left(1 / \sqrt{\lambda_{n}+1}\right) e^{n\left(\lambda_{n}+1 / \lambda_{n}-1\right)-1}\left(1+O\left(\lambda_{n} / n\right)\right),
$$

and

(iii) Maclaurin's expansion for $\lambda$ about $n$,

$$
\lambda_{n+j}=\lambda_{n}+\left(\lambda_{n} /\left(\lambda_{n}+1\right) n\right) j+O\left(j^{2} / n^{2}\right),
$$

we have that

$$
\left(\begin{array}{c}
n \\
i
\end{array}\right) \frac{B_{n-i}}{B_{n}}=\frac{1}{i !} \frac{n ! B_{n-1}}{(n-i) ! B_{n}}=\frac{1}{i !} e^{i\left[\ln n-\lambda_{n}+O\left(i^{2} / n\right)\right]}\left(1+O\left(\frac{\lambda_{n}}{n}\right)\right) .
$$

But $\lambda_{n} e^{\lambda_{n}}=n$ so $\ln n-\lambda_{n}=\ln \lambda_{n}$, and therefore

$$
\left(\begin{array}{c}
n \\
i
\end{array}\right) \frac{B_{n-i}}{B_{n}}=\frac{\lambda_{n}^{i}}{i !}\left(1+O\left(\frac{\lambda_{n}^{2}}{n}\right)\right)
$$

LEMMA 3. If $i=\sqrt{\lambda} s+\lambda$ then

$$
e^{-\lambda} \frac{\lambda^{l}}{i !}=\frac{1}{\sqrt{2 \pi}} e^{-s^{2} / 2}\left[1+\frac{s^{3}-3 s}{2 \sqrt{\lambda}}+\frac{s^{6}-12 s^{4}+27 s^{2}-6}{72 \lambda}+O\left(\frac{|s|^{9}}{\lambda^{3 / 2}}\right)\right] \text {. }
$$

Note that the first term of this series gives the classical approximation of the Poisson distribution by the normal. Its proof uses a stronger version 
of Stirling's formula $i !=\sqrt{2 \pi i}(i / e)^{i}\left(1+1 / 12 i+O\left(1 / i^{2}\right)\right)$ plus the series expansions for $e^{x}$ and $\ln (1+x)$.

\section{THEOREM 1.}

$\operatorname{Cov}\left(u_{s}^{(n)}, u_{t}^{(n)}\right)$

$$
\begin{array}{r}
=\frac{\delta(s, t)}{\sqrt{2 \pi}} e^{-s^{2} / 2}\left[1+\frac{s^{3}-3 s}{6 \gamma}+\frac{s^{6}-12 s^{4}+27 s^{2}-6}{72 \gamma^{2}}+O\left(\frac{|s|^{9}}{\gamma^{3}}\right)\right] \\
-\frac{1}{2 \pi} e^{-1 / 2\left(s^{2}+t^{2}\right)}\left[1+\frac{s^{3}+3 s+t^{3}+3 t}{6 \gamma}\right. \\
+\frac{s^{6}-9 s^{2}+2 s^{3} t^{3}+6 s^{3} t+6 s t^{3}+18 s t-84-9 t^{2}+t^{6}}{72 \gamma^{2}} \\
\left.+O\left(\frac{|s|^{9}+|t|^{9}}{\gamma^{3}}\right)\right] .
\end{array}
$$

Proof.

$$
\begin{aligned}
\operatorname{Cov}\left(u_{s}^{(n)}, u_{t}^{(n)}\right)=\frac{n}{\lambda^{2}}\left(\begin{array}{c}
n \\
i
\end{array}\right) \frac{B_{n-i}}{B_{n}}\left[\delta_{i, j}+\left(\begin{array}{c}
n-i \\
j
\end{array}\right) \frac{B_{n-t-j}}{B_{n}}-\left(\begin{array}{c}
n \\
j
\end{array}\right) \frac{B_{n-j}}{B_{n}}\right] & \text { by Lemma } 1 \\
= & \frac{n}{\lambda_{n}^{2}} \frac{1}{i !} \lambda_{n}^{i}\left(1+O\left(\frac{\lambda_{n}^{2}}{n}\right)\right)\left[\delta_{i, j}+\frac{1}{j !}\left(\lambda_{n-i}\right)^{j}\left(1+O\left(\frac{\lambda_{n-i}^{2}}{n}\right)\right)\right. \\
= & \left.\frac{n}{\lambda_{n}^{2}}\left[\frac{\lambda_{n}^{i}}{i !} \delta_{l, j}+\frac{1}{i ! j !} \lambda_{n}^{i+j}\left(\left(\frac{\lambda_{n-i}}{\lambda_{n}}\right)^{j}-1\right)\right]\left(1+O\left(\frac{\lambda_{n}^{2}}{n}\right)\right)\right] \\
= & \frac{n}{\lambda_{n}^{2}}\left[\frac{\lambda^{i}}{i !} \delta_{i, j}+\frac{1}{i ! j !} \lambda^{i+j}\left(\left(1-\left(\frac{\lambda_{n}^{2}}{n}\right)\right)\right.\right. \\
& \times\left(1+O\left(\frac{\lambda^{2}}{n}\right)\right) .
\end{aligned}
$$


By Maclaurin's expansion of $\lambda_{n-1}$ and then letting $\lambda=\lambda_{n}$,

$$
\begin{aligned}
& =\frac{n}{\lambda^{2}}\left[\frac{\lambda^{l}}{i !} \delta_{l, J}-\frac{\lambda^{l}}{i !} \frac{\lambda^{J}}{j !}\left(\frac{i j}{(\lambda+1) n}+O\left(\frac{\lambda}{n^{2}}\right)\right)\right]\left(1+O\left(\frac{\lambda^{2}}{n}\right)\right) \\
& =\frac{\delta(s, t)}{\sqrt{2 \pi}} e^{-s^{2} / 2}\left[1+\frac{s^{3}-3 s}{6 \gamma}+s \frac{s^{6}-12 s^{4}+27 s^{2}-6}{72 \gamma^{2}}+O\left(\frac{|s|^{9}}{\gamma^{3}}\right)\right] \\
& -\frac{1}{2 \pi} e^{-1 / 2\left(s^{2}+t^{2}\right)}\left[1+\frac{s^{3}+3 s^{2}+t^{3}+3 t}{6^{\gamma}}\right. \\
& +\frac{s^{6}-9 s^{2}+2 s^{3} t^{3}+6 s^{3} t+t s t^{3}+18 s t-84-9 t^{2}+t^{2}}{72 \gamma^{2}} \\
& \left.+O\left(\frac{|s|^{9}+|t|^{9}}{\gamma^{3}}\right)\right]
\end{aligned}
$$

by Lemma 3 .

COROllary. The distribution of the rank function $\tau(\sigma)=\sum_{i} \sigma_{i}$ is asymptotically Gaussian with mean

$$
\sum_{i} E\left(\sigma_{i}\right) \simeq \sum_{i} \frac{\lambda^{i}}{i !} \simeq e^{\lambda}=\frac{n}{\lambda}
$$

and variance

$$
\sum_{i, j}\left(\begin{array}{l}
n \\
j
\end{array}\right) \frac{B_{n-1}}{B_{n}}\left[\left(\begin{array}{c}
n-i \\
j
\end{array}\right) \frac{B_{n-i-j}}{B_{n}}-\left(\begin{array}{l}
n \\
j
\end{array}\right) \frac{B_{n-j}}{B_{n}}\right]=\frac{1}{\lambda}+O\left(\frac{1}{\gamma^{3}}\right) .
$$

This has already been proven by myself (in [6]) and E. Bender.

III. A continuous analog of Sperner's problem. The discussion in the preceding section suggests that we consider the effect which asymptotic normality has on a weighted Sperner problem: Assume that the limit Gaussian distribution on $\mathbf{R}^{n}$ has mean 0 and covariance matrix $C$. The weight then is the Gaussian density function

$$
f(x)=\frac{1}{(\sqrt{2 \pi})^{n} \operatorname{det}(C)} e^{-1 / 2\left(x C^{-1} x^{t}\right)} .
$$

Assume also that the limit partial order $P$ on $\mathbf{R}^{n}$ is determined by a cone $K \subseteq \mathbf{R}^{n}$ with rank function $r(x)=x \cdot e, e \in K$.

EXAMPLES. (i) If $e$ is the unit vector in a direction which maximizes the quadratic form $x C x^{t}$, a little reflection shows that the hyperplane 
$P_{0}=\{x: x \cdot e=0\}$ is a set of incomparables and has the maximum weight of any hyperplane. (ii) Suppose

$$
C=\left(\begin{array}{ccc}
\sigma_{1}^{2} & & 0 \\
& \ddots & \\
0 & & \sigma_{n}^{2}
\end{array}\right)
$$

a diagonal matrix, and $K=\left\{\sum_{i=1}^{n} \alpha_{i} \delta_{i}: \alpha_{l} \geq 0\right\}$, the positive orthant in $\mathbf{R}^{n}$. Then $P$ is a product weighted poset, each factor being Gaussian and therefore $\log$ convex. The continuous analog of the Product Theorem ([7]) would imply the strong Sperner property for $P$.

Definition. Given a continuous (Gaussian) weighted partial order $P$ on $\mathbf{R}^{n}$ determined by a covariance matrix $C$, cone $K \subseteq \mathbf{R}^{n}$ and rank function $r(x)=x \cdot e$, call $d=C e^{t}$ its direction.

TheOREM 2. If $d \in K$ then $P$ has the (strong) Sperner property.

Note. Looking at the preceding examples in the light of this theorem we see that in

(i) $d=C e^{t}=\lambda e$ since $e$ is an eigenvector of $C$, and since $e \in K$, $d \in K$, and in

(ii) $d=C e^{t} \geq 0$ so $d \in K$. In both examples then the hypothesis of Theorem 2 is satisfied and so $P$ has the Sperner property.

Proof. Again, this proof is the conjunction of two insights:

(i) If a weighted poset $P$ can be partitioned into chains on each of which the weights are arranged in the same relative order (maximum on $P_{0}$, etc.) then $P$ has the strong Sperner property, and

(ii) For $x \in \mathbf{R}^{n}$ such that $x \cdot e=r$ let $x-(r /(d \cdot e)) d=x_{0}$. Then $x_{0} \in P_{0}$ and $x=x_{0}+(r /(d \cdot e)) d$ so

$$
x C^{-1} x^{t}=x_{0} C^{-1} x_{0}^{t}+\left(\frac{r}{d \cdot e}\right)^{2}\left(d C^{-1} d^{t}\right)
$$

since $x_{0} C^{-1} d=x_{0} C^{-1}(C e)=x_{0} \cdot e=0$. Therefore

$$
\begin{aligned}
f(x) & =\frac{1}{(\sqrt{2 \pi})^{n} \operatorname{det} C} e^{-1 / 2\left(x C^{-1} x^{t}\right)} \\
& =\left(\frac{e^{-1 / 2\left(x_{0} C^{-1} x_{0}^{t}\right)}}{(\sqrt{2 \pi})^{n} \operatorname{det} C}\right) \exp \left(-\frac{d C^{-1} d^{t}}{2(d \cdot e)^{2}} r^{2}\right) .
\end{aligned}
$$


Noting that the first factor in $f(x)$ depends only upon $x_{0}$ and the second only upon $r$, we see that if $d \in K$ then $\left\{x_{0}+(r /(d \cdot e)) d: r \in \mathbf{R}\right\}$ is a chain and the condition of part $i$, is satisfied.

IV. Is $d \in K$ for $\Pi_{n} / \mathbf{S}_{n}$ ? Assuming that Theorem 2 for Gaussian distributions on $\mathbf{R}^{n}$ carries over to Gaussian processes, we calculate the direction

$$
\begin{aligned}
d_{\gamma}(s) & =\int_{-\infty}^{\infty} \operatorname{Cov}\left(u_{s}^{(n)}, u_{t}^{(n)}\right) d t \\
& =\frac{1}{\gamma \sqrt{2 \pi}} e^{-s^{2} / 2}\left[-s+\frac{-s^{4}+3 s^{2}+6}{6 \gamma}+O\left(\frac{|s|^{2}}{\gamma^{2}}\right)\right]
\end{aligned}
$$

and we want to know if this function is in the cone

$K_{\gamma}=\left\{\int_{-\infty}^{\infty} \int_{-\infty}^{\infty} \alpha(x, y)\left[\delta_{x}(s)+\delta_{y}(s)-\delta_{x+y+\gamma}(s)\right] d x d y \mid \alpha(x, y) \geq 0\right\}$.

In order to answer this question we must introduce the notion of the dual of a cone. If $K \subseteq \mathbf{R}^{n}$ is a cone then define

$$
K^{*}=\left\{y \in \mathbf{R}^{n} \mid \forall x \in K, x \cdot y \geq 0\right\} .
$$

EXAMPLES. (i) If $K$ is a half-line then $K^{*}$ is a half-space and vice versa.

(ii) The positive orthant $\left(\mathbf{R}^{n}\right)^{+}$is self-dual.

The Duality Theorem. (See [5].) If $K$ is a closed cone then $\left(K^{*}\right)^{*}=K$.

Note. The duality theorem may not hold in function spaces but certainly $K \subseteq K^{* *}$. Also $K \subseteq J \Rightarrow J^{*} \subseteq K^{*}$.

Extending this definition to our infinite dimensional space we have $f \in K_{\gamma}^{*}$ iff $f \cdot\left(\delta_{x}+\delta_{y}-\delta_{x+y+\gamma}\right) \geq 0 \quad \forall x, y \in \mathbf{R}$, i.e. $f(x)+f(y) \geq$ $f(x+y+\gamma)$. When $\gamma=0$ these are called subadditive functions.

LEMma 4. $f(x) \in K_{\gamma}$ iff $f(x+\gamma) \in K_{0}$.

Proof. If $g(x)=f(x+\gamma)$ then

$$
g(x)+g(y)=f(x+\gamma)+f(y+\gamma) \geq f(x+y+\gamma)=g(x+y),
$$
so $g \in K_{0}$ and conversely.

Note. An obvious subclass of $K_{0}^{*}$ to study is the additive functions (those for which equality always holds). It is well known (see [1]) that the 
only measurable additive functions are the linear functions $y=c x$. Another subclass of $K_{0}^{*}$ (in fact of any $K_{\gamma}^{*}$ ) is the functions $f \geq 0$ such that $\lim \sup (f) \leq 2 \liminf (f)$. Then

$$
f(x)+f(y) \geq 2 \lim \inf (f) \geq \lim \sup (f) \geq f(x+y+\gamma) .
$$

THEOREM 3. For $n$ sufficiently large $\left(6 \times 10^{6}\right.$ should do it $) d_{\gamma} \notin K_{\gamma}$.

Proof. For any $A \subseteq \mathbf{R}$, let

$$
X_{A}(s)= \begin{cases}1 & \text { if } s \in A \\ 0 & \text { if } s \notin A .\end{cases}
$$

By the note above $1+X_{A} \in K_{\gamma}^{*}$. If we can find an $A$ such that

$$
d_{\gamma} \cdot\left(1+X_{A}\right)=\int_{-\infty}^{\infty} d_{\gamma}(s)\left(1+X_{A}(s)\right) d s<0
$$

this would imply $d_{\gamma} \notin K_{\gamma}$. Now with $A=\{s \geq 0\}$ this holds for $n$ sufficiently large since $\int_{-\infty}^{\infty} d_{\gamma}(s) d s=1 / \gamma$ and $\int_{0}^{\infty} s e^{-s^{2} / 2}=1$. To get a better estimate of the smallest $n$ for which $d_{\gamma} \notin K_{\gamma}$, we note that $d_{\gamma}(s)<0$ for $s$ sufficiently large. We thus let $A=\left\{s \geq s_{0}\right\}$ and find the simultaneous solution of

$$
d_{\gamma}\left(s_{0}\right)=0, \quad \int_{s_{0}}^{\infty} d_{\gamma}(s) d s=-1 / \gamma
$$

By Newton's method we find $\gamma=3.62$ which gives $n=\gamma^{2} e \gamma^{2} \simeq 6 \times 10^{6}$.

Note. The preceding discussion does not demonstrate that $d_{\gamma} \in K_{\gamma}$ for $n \leqslant 6 \times 10^{6}$, but strongly suggests it: If we had a good characterization of the extreme points of $K_{0}^{*}$ and if Farka's Lemma extended to show $K_{\gamma}^{* *}=K_{\gamma}$, then the above computation could be sharp. Peck's improvement $\left(5.5 \times 10^{6}\right)$ of Canfield's example is also close enough to provide support for this idea.

V. The objective function. Since $d_{\gamma} \notin K_{\gamma}$ for $n$ sufficiently large and we felt that this condition is essentially equivalent to the Sperner property, we look for sets of incomparables larger (in weight) than $P_{0}$. In order to do this we must better understand the objective function of our continuous Sperner problem. If we restrict our sets of incomparables in $\mathbf{R}^{n}$ to be smooth hypersurfaces (of dimension $n-1$ ) then reflection shows the objective function to be of the form $F(I)=\int_{I} f(x) g_{I}(x) d A_{I}(x)$ where $f$ is the Gaussian density function,

$$
f(x)=\frac{1}{(\sqrt{2 \pi})^{n}} \operatorname{det}(C) e^{-1 / 2\left(x C^{-1} x^{t}\right)},
$$


$g_{I}$ is a geometrical factor and the integration is with respect to the area of $I$ near $x$. If $I$ is further restricted to be a hyperplane (and we may assume $0 \in I$ ) then $g_{I}$ is constant as is the local area. Therefore $F(I)=$ $g_{I} \int_{I} f(x) d A(x)$ and by direct calculation $\int_{I} f(x) d A(x)=1 / \sqrt{2 \pi u C u^{t}}$ where $u$ is the unit normal to $I$, i.e. $I=\{x: x \cdot u=0\}, u \cdot u=1$ and $u \cdot e>0$.

Lemma 5. A hyperplane $H=\{x \cdot u=0\} \subseteq \mathbf{R}^{n}\left(\mathbf{R}^{n}\right.$ partially ordered by a cone $K)$ is a set of incomparables iff $u$ is in the interior of $K^{*}$.

Proof. Suppose $y \neq x$ then $x, y \in H \Rightarrow y-x \in H \Rightarrow(y-x) \cdot u=$ 0 . Also $x<y \Leftrightarrow y-x \in K$. Therefore, if $H$ is not a set of incomparables then $u$ is not in the interior of $K$. Conversely, if $H$ is a set of incomparables and $y \in K, y \neq 0$ then $y \cdot u \neq 0$. We assume that $e \in K$ and $u \cdot e>0$. If $y \cdot u<0$ then there would exist $0<\lambda<1$ such that $(\lambda e+(1-\lambda) y)$. $u=0$ so $\lambda e+(1-\lambda) y \in H \cap K$ which is a contradiction. Therefore $y \cdot u>0$ and $u$ is in the interior of $K^{*}$.

Thus our modified Sperner problem is to find

$$
\max _{\substack{u \in K^{*} \\\|u\|=1}} \frac{g(u)}{\sqrt{2 \pi u C u^{t}}} .
$$

Note. The geometric factor $g$ must be continuous as a function of $u$, but Theorem 2 implies that its derivative is discontinuous at $u=e$.

In order to increase our undertanding of the geometric factor, let us examine a specific example: Let $n=2, K$ be the positive quadrant in $\mathbf{R}^{2}$ and $e=(1 / 2,1 / 2)$ but take $0 \leq \Theta \leq \pi / 4$ to be the parameter determining the hyperplane $H$ rather than $u$. (See Figure 1.) $g(\Theta)$ is then the density of incomparable lattice points which lie on or near the hyperplane

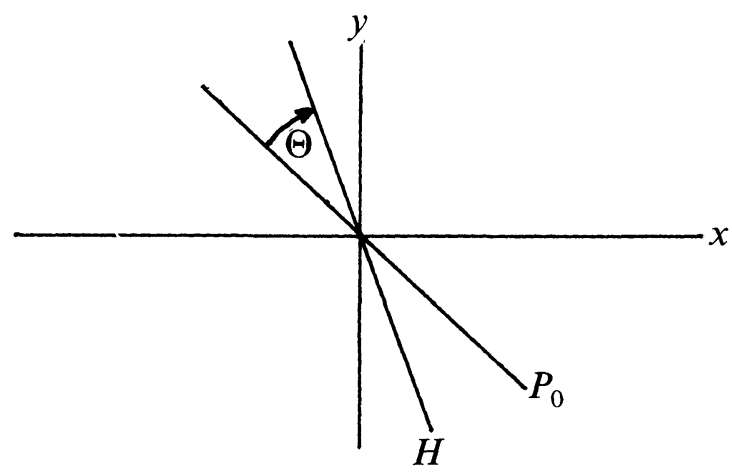

FigURE 1 
(line) $H$. The density of candidate points near $H$ is $\sin (\pi / 4+\Theta)$ but one of these must be eliminated each time the rank increases (see Figure 2) so we have

$$
g(\Theta)=\sin (\pi / 4+\Theta)\left(1-\frac{1}{m}\right)
$$

where $m$ is determined by $\tan \Theta=1 /(2 m-1)$. Therefore

$$
m=1 / 2\left(1+\frac{1}{\tan \Theta}\right) \text { and } 1-\frac{1}{m}=\frac{\cos \Theta-\sin \Theta}{\cos \Theta+\sin \Theta} .
$$

Finally

$$
g(\Theta)=\left(\frac{\cos \Theta+\sin \Theta}{\sqrt{2}}\right) \frac{\cos \Theta-\sin \Theta}{\cos \Theta+\sin \Theta}=\frac{\cos \Theta-\sin \Theta}{\sqrt{2}} .
$$

By symmetry $g(-\Theta)=g(\Theta)$ and we see that $g^{\prime}$ is indeed discontinuous at $\Theta=0$.

In general we have

LeMma 6. $g(u)=\min _{x \in K}(x \cdot u) /(x \cdot e)$

In order to derive this expression for $g$ we extend the notion of a set of incomparables: A fat hyperplane is the union of contiguous translates of

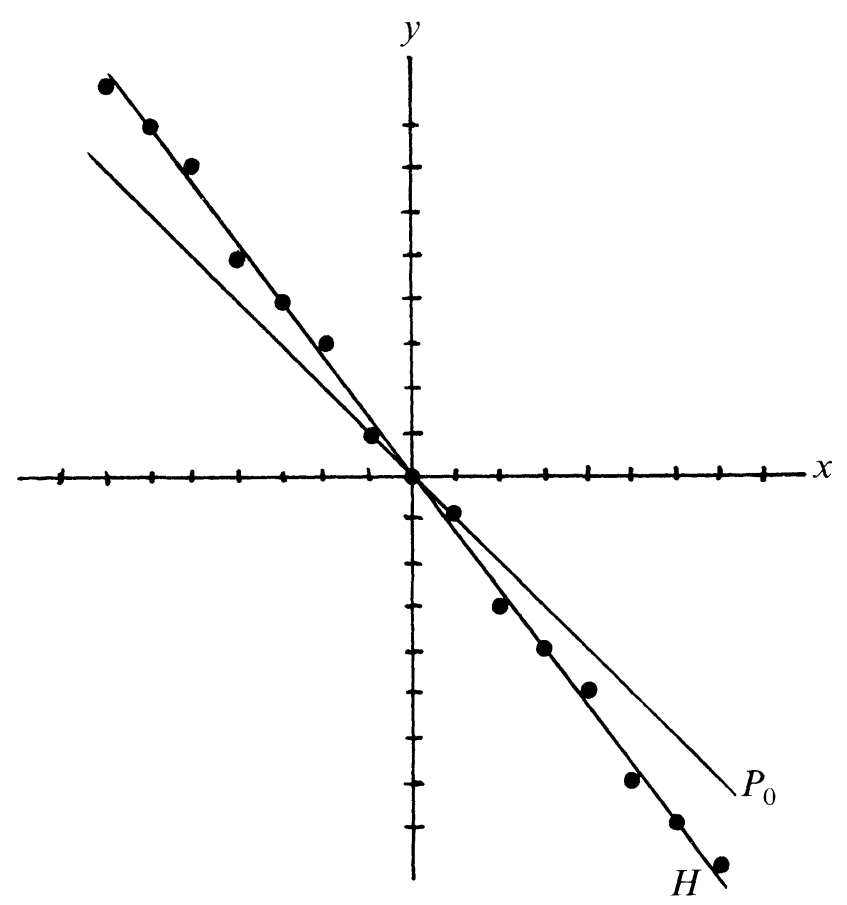

FigURE 2 
a given hyperplane. The maximum distance between any point and its orthogonal translate is the thickness of the fat hyperplane. A fat hyperplane for which the maximum difference in rank between two comparable members is $l$ is called $l$-Sperner. A 0 -Sperner set is then a set of incomparables.

Proof. Let a fat hyperplane $H$ with unit normal $u$ and thickness $\tau$ be $l$-Sperner for some $l>0$. We may assume that 0 is contained in the lower boundary of $H$. Then for all $x \in H \cap K$ if $x$ is contained in the upper boundary of $H$ we have

$$
x \cdot u=\tau \text { and } x \cdot e \leq l .
$$

Combining these we have

$$
\tau=(x \cdot u) \times 1 \leq \frac{x \cdot u}{x \cdot e} l .
$$

But the r.h.s. is independent of the length of $x$ so

$$
\tau \leq\left(\min _{x \in K}((x \cdot u) /(x \cdot e))\right) l .
$$

This means that $g \leq \min _{x \in K}((x \cdot u) /(x \cdot e))$ and, since this is the only restriction on $g$, equality holds.

VI. The asymptotic Rota conjecture. If we simplify the objective function of the previous section by squaring and then deleting constant factors we have $G(u) / F(u)$ where $G(u)=\left(\min _{x \in K}((x \cdot u) /(x \cdot e))\right)^{2}$ and $F(u)=u C u^{t}$. This is to be maximized over all $u \in K^{*}$ such that $\|u\|=1$. This can be further simplified by noting that $\min _{x \cdot e=1 ; x \in K}(x \cdot u)$ is a linear program so we may assume that $x$ is an extreme point of $K \cap\{x \cdot e=1\}$. Also $G(\lambda u) / F(\lambda u)=G(u) / F(u)$ so we may drop the condition that $\|u\|=1$.

Since $G(e)=1$, the asymptotic Rota conjecture is equivalent to $F(e) \max _{u \in K^{*}}(G(u) / F(u)) \rightarrow 1$ for $\Pi_{n} / \mathbf{S}_{n}$ as $n \rightarrow \infty$. However, if we take $u=e-\varepsilon \bar{d}$ where $\varepsilon>0$ and $\bar{d}=\gamma d_{\gamma}$, then $x \cdot u=x \cdot(e-\varepsilon \bar{d})=1$ $-\varepsilon(x \cdot \bar{d})$ and $G(e-\varepsilon \bar{d})=\left(\min _{x \in K ; x \cdot e=1}(1-\varepsilon(\bar{d}))^{2}=(1-\varepsilon M(\gamma))^{2}\right)$ where $M(\gamma)=\max _{x \in K ; x \cdot e=1} x \cdot \bar{d}$. Since $x$ may be assumed an extreme point of $K \cap\{x \cdot e=1\}$,

$$
\begin{aligned}
M(\gamma) & =\max _{s, t}\left(\left(\delta_{s}+\delta_{t}-\delta_{s+t+\gamma}\right) \cdot \bar{d}\right) \\
& =\max _{s, t}(\bar{d}(s)+\bar{d}(t)-\bar{d}(s+t+\gamma)) \rightarrow 2 \max _{s} \bar{d}(s) \text { as } n \rightarrow \infty \\
& =\sqrt{2 / \pi e} .
\end{aligned}
$$


By similar reasoning and the note after Lemma 4 , if $\varepsilon \leq \sqrt{2 \pi e} / 3$ then $e-\varepsilon \bar{d} \in K^{*}$.

Now

$$
\begin{aligned}
\frac{F(e)}{F(e-\varepsilon \bar{d})} & =\frac{e C e^{t}}{(e-\varepsilon \bar{d}) C(e-\varepsilon \bar{d})^{t}} \\
& =\frac{1}{1-2 \varepsilon(\bar{d} \cdot \bar{d}) /(e \cdot \bar{d})+\varepsilon^{2} \gamma \bar{d} C \bar{d}^{t} /(e \cdot \bar{d})} .
\end{aligned}
$$

By direct computation

$$
\begin{aligned}
& e \cdot \bar{d}=\frac{1}{\gamma}+O\left(\frac{1}{\gamma^{2}}\right)=\frac{1}{\gamma}\left(1+O\left(\frac{1}{\gamma}\right)\right) \\
& \bar{d} \cdot \bar{d}=\frac{1}{4 \sqrt{\pi}}+O\left(\frac{1}{\gamma}\right) \text { and } \bar{d} C \bar{d}^{t}=\frac{1}{6 \pi \sqrt{3}}+O\left(\frac{1}{\gamma}\right),
\end{aligned}
$$

so

$$
\begin{aligned}
\frac{F(e)}{F(e-\varepsilon \bar{d})} & =\frac{1}{1-(\varepsilon \gamma)(1 / 2 \sqrt{\pi}+O(1 / \gamma))+(\varepsilon \gamma)^{2}(1 / 6 \pi \sqrt{3}+O(1 / \gamma))} \\
& =\frac{1}{1-3 \sqrt{3} / 8+O(1 / \gamma)} \quad \text { if } \varepsilon=\frac{3 \sqrt{3 \pi}}{2 \gamma}=2.85 \ldots+O\left(\frac{1}{\gamma}\right) .
\end{aligned}
$$

Since $\varepsilon=O(1 / \gamma)$, the geometric factor $G(n-\varepsilon \bar{d})=1+O(1 / \gamma)$ is negligable and the asymptotic Rota conjecture is false (though not by all that much!).

VII. Comments and conclusions. Much remains to be done to bring the material in $\S \S I-V I$ of this paper up to twentieth century standards of rigor, but I am fairly confident that the essentials of the argument are correct. Primary among the logical gaps is the assumption that $\Pi_{n} / \mathbf{S}_{n}$ may be approximated by a partially ordered Gaussian process. Clearly the notion of convergence required will be a variant of weak convergence from probability theory (see [3]), but there are a number of complicating factors:

(i) The parameter $1 / \gamma$ may either be considered an infinitesimal, characteristic of the distribution of the limit process, $u_{s}$, or it may assume finite values specifying the covariance of finite dimensional Gaussian processes co-convergent with the seqeunce $u_{s}^{(n)}$.

(ii) A strong type of weak convergence is required since we wish to replace maximizing the weight of sets of incomparables in $\Pi_{n} / \mathbf{S}_{n}$ by 
maximizing the integral $\int_{I} f(x) g_{I}(x) d A(x)$ over sets of incomparables, $I$ in a partially ordered vector space.

(iii) Difficulties (i) and (ii) are compounded by the subtlety of the computations. In computing $d_{\gamma}$ the lowest order term drops out and in the counterexample to the asymptotic Rota conjecture letting $\varepsilon$ approach 0 in the prescribed way gives a different result from letting $\varepsilon$ equal 0 .

(iv) The convergence of partial orders must be justified. The way in which we passed from discrete to continuous posets seems natural but requires study.

Another logical gap apparently occurs when we assume that results proven for finite dimensional continuous Sperner problems (e.g. Theorem 2) apply to infinite dimensional ones. This difficulty is avoided to a certain extent if we choose to work with co-convergence in part (i) of the preceding paragraph, but we must still wrestle with the relationship between $K_{\gamma}, K_{\gamma}^{*}$ and their finite dimensional counterparts.

Besides these logical gaps there are some computational problems which remain unsolved:

(i) We need a characterization of the extreme points of $K_{0}^{*}$, the cone of all subadditive functions. Subadditive functions have been studied by functional analysts, but no information on their extreme points beyond what is in the note after Lemma 4 seems to be available.

(ii) An exact solution of the continuous Sperner problem would be a great help in pinning things down. Is the solution necessarily a surface and if so does it have to be a hyperplane? Given that the answers to these two questions are yes, I think that the asymptotic ratio of $\sqrt{2.85} \simeq 1.69$ is the best possible, but if not it could go considerably higher.

\section{REFERENCES}

[1] J. Aczel, Lectures on Functional Equations and their Applications, Academic Press, New York, 19966.

[2] E. A. Bender, Central and local limit theorems applied to asymptotic enumeration, J. Combinatorial Theory-Ser. A, 15 (1977), 91-111.

[3] P. Billingsley, Convergence of Probability Measures, Wiley, New York, 1968.

[4] E. R. Canfield, On a problem of Rota, Advances in Math., 29 (1978), 1-10.

[5] M. Hall, Jr., Combinatorial Theory Ginn (Blaisdell), Waltham, Mass., 1967.

[6] L. H. Harper, Stirling behavior is asymptotically normal, Ann. Math. Stat., 38, No. 2 (1967), 410-414.

[7] The morphology of partially ordered sets, J. Combinatorial Theory, 17, No. 1 (1974), 44-57.

[8] The global theory of flows in networks, Advances in App. Math., 1 (1980), $158-181$.

[9] J. M. De Laurentis and B. G. Pittel, Counting subsets of the random partition and the “Brownian bridge" process, Stochastic Process. Appl., 15 (1983), 155-167. 
[10] L. Moser and M. Wyman, An asymptotic formula for the Bell numbers, Trans. Royal Soc. Canada, 49, Ser. 3 (1955), 49-51.

[11] G. C. Rota, Research problem: “A generalization of Sperner's theorem, J. Combinatorial Theory, 2 (1967), 104.

[12] J. B. Shearer, A simple counterexample to a conjecture of Rota, Discrete Math., 28 (1979), 327-330.

[13] J. H. Spencer, A generalized Rota conjecture for partitions, Studies in Appl. Math., 53 (1974), 239-241.

[14] E. Sperner, Ein Satz uber Untermengen einer endlichen menge, Math. Z., 27 (1928), 544-548.

Received October 11, 1984.

UNIVERSITY OF CALIFORNIA

RIVERSIDE, CA 92521 
